\section{GRUPOS COM CUIDADORES DE PESSOAS COM DOENÇA DE PARKINSON (DP): UM CONVITE À REFLEXÃO}

\author{
GROUPS WITH CAREGIVERS OF PEOPLE WITH PARKINSON'S DISEASE (PD): \\ AN INVITATION TO REFLECTION
}

\begin{abstract}
RESUMO: 0 artigo convida à reflexão sobre a importância dos diálogos em grupos de pessoas com (DP) e com seus cuidadores. Estes são grupos de pessoas com limitações sociais, devido aos efeitos do processo de envelhecimento, da DP e, no caso dos cuidadores, da atenção ao paciente. A reflexão proposta é fruto de diversas ações de pesquisa e de extensão, com destaque para o relato sobre grupos de partilha com cuidadores de pessoas com DP, que com o objetivo de gerar espaços de trocas e de atenção aos cuidadores, resultaram em reflexões relevantes. Os principais temas, sugeridos pelos cuidadores: sobrecarga do cuidador/a familiar, relação cuidador/a-pessoa com DP, práticas que promovam o cuidado, o desenvolvimento, a convivência, o lazer e a autonomia dos participantes. 0 grupo de partilha é parte de um projeto de extensão interistitucional e transdisciplinar, e está embasado em pesquisas de cunho sistêmico sobre a rede social pessoal (Sluzki, 1997).
\end{abstract}

Palavras-chave: Grupos; Cuidadores; Doença de Parkinson.

ABSTRACT: The article invites to reflect on the importance of dialogues in groups of people with PD and with caretakers for people with PD. These are groups of people with social limitations due to the effects of the aging process, PD and, in the case of caregivers, the patient care. The proposed reflection is a product of multiple research and extension projects, with emphasis on the report on sharing groups with caretakers of people with PD, that aim to make space for exchange and attention to caretakers and result in relevant reflections. The main themes suggested by caretakers on the group, were: overworking the family caretaker, the caretaker-person with PD relationship, practices that promote better care, development, coexistence, leisure and participant autonomy. The sharing group is part of an interistitutional, and transdisciplinary extension project, is based on systemic researches about the social network (Sluzki, 1997).

Keywords: Groups; Caregivers; Parkinson's Disease.

RESUMEN: El artículo invita a reflexionar sobre la importancia de los diálogos en grupos de personas con EP y con cuidadores. Estos son grupos de personas con limitaciones sociales debido a los efectos del proceso de envejecimiento, la EP y, en el caso de los cuidadores, la atención al paciente. La reflexión propuesta es producto de múltiples proyectos de investigación y extensión, con énfasis en el informe de grupos con cuidadores de personas con EP, que tienen como objetivo hacer espacio para el intercambio y atención a los cuidadores. Los temas principales sugeridos por los cuidadores fueron: sobrecargar al cuidador familiar, el cuidador-persona con relación de EP, prácticas que promueven una mejor atención, desarrollo, convivencia, ocio y autonomía de los participantes. El grupo para compartir es parte de un proyecto de extensión interistitutional, transdisciplinario, se basa en encuestas sistémicas sobre la importancia de la red social (Sluzki, 1997).

Palabras clave: grupos; cuidadores; Enfermedad de Parkinson.

\section{MARIANNE RAMOS FEIJÓ 1}

FABIO AUGUSTO

BARBIERI $^{1}$

\section{TAFNES}

IKEGAMI PEREIRA ${ }^{1}$

\section{BIANCA SILVA AUGUST0 1}

\section{CAROLINA} SILVA GASPAR ${ }^{2}$

${ }^{1}$ Universidade Estadual Paulista (UNESP),

Bauru, SP, Brasil

2 Tribunal de Justiça do Estado de São Paulo, Brasil

Recebido em 11/02/2020 Aprovado em 18/11/2020 


\section{INTRODUÇÃO}

A reflexão sobre a importância dos espaços de diálogo com grupos de pessoas com DP e com grupos de cuidadores de pessoas com DP partiu de uma experiência prática em andamento, o Grupo de Partilha (GP), criado com o objetivo inicial de gerar troca entre cuidadores familiares de idosos que esperavam as pessoas de quem cuidam realizarem atividades físicas, de avaliação e de socialização, oferecidas em um projeto de extensão, do qual a maior parte dos autores faz parte. Trata-se de um projeto de extensão voltado à promoção de saúde e de qualidade de vida de pessoas com DP, o que, por sua vez, depende do bem-estar (ou do estar bem) dos seus cuidadores, que antes do início do GP compareciam ao projeto apenas como acompanhantes de pessoas com redução de habilidades motoras e do equilíbrio. A maioria dos participantes do projeto de extensão e daqueles que se apresentam como a pessoa da família que cuida é formada por idosos. O GP, grupos quinzenais com propósitos de fomentar trocas de experiências e de informações e de refletir sobre o cuidar, tem sido realizado com os cuidadores, cujas dificuldades como sobrecarga emocional são acolhidas e discutidas com mediação de estagiárias de psicologia. Há um ano, o grupo de cuidadores foi convidado a se reunir para dialogar sobre a experiência de cuidar e a escolher temas do seu interesse para discussão e reflexão. A busca por informações corretas sobre a doença, sintomas, cuidados e tratamentos também foi um compromisso assumido pelas mediadoras do grupo, que em contato com cuidadores e com a equipe de profissionais do projeto, quinzenalmente, conectavam necessidades e informações. A participação nos referidos grupos e a supervisão de atividades de extensão por dois dos autores permitiram observar que, dentre os temas mais abordados pelos cuidadores, figura a importância da rede social significativa tanto para eles, que já participam dos GP, como das pessoas com DP, a quem serão oferecidos grupos no próximo semestre. A reflexão de que a rede deve ser preservada e ampliada, especialmente no caso de idosos, como já apontou Sluzki (1997), se aplica a pessoas com dificuldades de locomoção, como as pessoas com DP, cujos contatos sociais tendem a ser reduzidos, e aos cuidadores em tempo integral, cuja qualidade de vida e saúde afeta também a relação com quem cuidam e com os demais familiares.

O bom relacionamento com pessoas significativas, o acesso e o suporte da rede social são considerados fatores de resiliência, portanto, de proteção à saúde humana. Pessoas com sessenta anos ou mais, cujas oportunidades de deslocamento e de convivência podem ser reduzidas progressivamente, necessitam de outras pessoas, com as quais convivam bem e em quem tenham confiança, para que seja mantida sua qualidade de vida (Brito \& Pavarini, 2012; Couto, Koller, \& Novo, 2006; Dias, Carvalho \& Araújo, 2013; Feijó, 2006). Os grupos de partilha com cuidadores de pessoas com DP têm sido um espaço de reflexão importante sobre o cuidar, sobre as relações significativas e atividades de lazer para o/a cuidador/a e para a pessoa com DP, que, quando diversificadas e ampliadas, reduzem a sobrecarga e promovem qualidade de vida.

Sluzki (1997) estudou a rede social pessoal e a saúde e descreveu algumas das funções da rede social, tais como: (a) companhia emocional - compreensão, simpatia, empatia, estímulo e apoio, função típica das amizades íntimas e das relações familiares próximas; guia cognitivo e de conselhos; (b) regulação (ou controle) social - intercâmbios que enfatizam responsabilidades e neutralizam os desvios de comportamento; (c) ajuda material e de serviços - indicação de serviços como os de saúde, além de ajuda financeira e física; (d) acesso a novos contatos relações que promovem a conexão com pessoas e redes novas para o indivíduo.

Nova Perspectiva Sistêmica, v. 29, n. 68, p. 31-45, dezembro 2020. 
Para o referido autor, uma pessoa pode desempenhar diversas funções, o que ocorre com frequência entre as pessoas mais íntimas, cujo afastamento traz vulnerabilidade principalmente aos idosos - já que a rede social significativa destes tende a encolher por morte de pessoas da mesma geração (Sluzki, 1997). No acompanhamento de pacientes idosos com DP, os autores observam que algumas pessoas limitam bastante o círculo social e as atividades de lazer com o avanço de fase da DP. Os cuidadores familiares, por sua vez, frequentemente centram sua vida no cuidar, o que também reduz a sua rede social. Tal redução de convivência, pré-institucionalização dos idosos, foi estudada por Gaspar e Hernandes (2016).

Brito e Pavarini (2012) correlacionaram apoio emocional e capacidade funcional de idosos em uma pesquisa realizada com 101 idosos com alterações cognitivas, cadastrados em Unidades de Saúde da Família. Os idosos e pessoas que, acometidas por alguma doença, dependem de cuidados durante todo o seu dia, podem ter na figura do/a cuidador/a uma fonte de apoio e de estímulo. Para isso, o/a cuidador/a deve estar preparado, orientado, disponível e também ciente de seu papel na manutenção da autonomia e do bem-estar tanto de si mesmo quanto da pessoa que cuida. Não se trata de uma tarefa fácil, mas de um grande desafio, impactado por inúmeros fatores: condições econômicas, progresso da doença, disponibilidade de outros cuidadores e acesso às pessoas e instituições relevantes para ambos (família, serviços de saúde qualificados, espaços de lazer). Cabe ressaltar que o cuidado com o/a cuidador/a é fundamental no referido processo (Grandesso \& Barreto, 2007; Horta \& Daspett, 2007). Do contrário, suas forças podem se esgotar e empobrecer a atenção e convivência com a pessoa que necessita do cuidado, situação que pode se caracterizar por negligência e violência em casos extremos (Grossi \& Santos, 2016).

Pautado em atividades de extensão (Moretto et al., 2015) e em pesquisas sobre a rede social de idosos (Gaspar \& Hernandes, 2016) e sobre o preparo de cuidadores de pessoas com DP (Augusto \& Feijó, 2019), no presente artigo visa-se à reflexão sobre a importância de grupos de trocas para cuidadores e para as pessoas diagnosticadas com doença de Parkinson (DP), que incluam diálogos e reflexões sobre temas por eles escolhidos. Cabe ressaltar que muitos cuidadores são também familiares com mais de 60 anos que precisam manter atividades de convivência para além do cuidar. Do contrário, estarão sujeitos à redução de rede social de suporte e ao esgotamento de recursos emocionais para lidar com a delicada tarefa de colaborar com a manutenção da qualidade de vida e da autonomia da pessoa em fases mais avançadas da DP.

De uma forma geral, com o passar dos anos, a rede social dos idosos tende a se contrair. Isso acontece devido a maior ocorrência de morte de pessoas da mesma geração e de seus antecessores, de dificuldades de locomoção, de acuidade visual, de transporte e de autonomia, além de escassez de situações sociais que oportunizam novas amizades na escola, no trabalho ou em viagens (Sluzki, 1997). Quando uma pessoa recebe o diagnóstico de DP e passa a ser cuidada por outra pessoa da família, ambas podem ter reduzidas sua vida social e as trocas afetivas. Os cuidadores profissionais também devem evitar a sobrecarga com o trabalho e a redução de outras atividades sociais e de lazer, mas na experiência dos autores, são familiares os participantes dos GP. Segundo Sluzki (p. 41), a rede social pessoal, definida pelas "relações que um indivíduo percebe como significativas ou define como diferenciadas da massa anônima da sociedade", constitui um importante fator para a promoção da saúde. Desta forma, refletir com familiares cuidadores e com as pessoas com DP sobre seus laços e atividades de convivência é importante ação de cuidado para com os mesmos, inclusive para que sejam minimizados episódios de depressão, que podem estar associados ao isolamento e à falta de suporte social 
íntimo (Dias, Carvalho, \& Araújo, 2013; Marinho, 2010; Rizzolli \& Surdi, 2010; Saintrain, Bandeira, Nobre \& Sandrin, 2018; Vitorino, Paskulin \& Vianna, 2013).

Ainda sobre a relevância do trabalho com cuidadores de pessoas com DP em grupos formados por muitos idosos, em 2014, a proporção da população idosa brasileira atingiu a marca de 13,7\% (Giacomin, 2016). As cidades, os serviços de saúde, as famílias e os cuidadores raramente estão preparados para a garantia do acesso a direitos, a promoção da autonomia e da qualidade de vida da população que, por avanço da idade e/ou a presença de deficiência e de certas disfuncionalidades, depende de um cuidado específico e da quebra de barreiras à sua inclusão (Camargo \& Feijó, 2017). O Censo apontou que mais de 23\% da população apresenta alguma deficiência, e segundo o site do IBGE (2019), mais de 200 mil pessoas apresentavam DP em 2017. Entre 2005 e 2015, a proporção de idosos de 60 anos ou mais na população do Brasil passou de 9,8\% para 14,3\% e poderá chegar a mais de 35\% em 2017.

De acordo com a Aliança de Cuidadores Familiares, ou Family Caregivers Alliance (Friedland, 2019), 12 milhões de americanos precisavam de cuidados prolongados em 2007 e, com o contínuo crescimento da população de idosos, até 2050, o número de pessoas que utilizam serviços pagos de assistência de longo prazo em qualquer ambiente (por exemplo, em casa, atendimento residencial, como moradia assistida ou instalações de enfermagem especializadas) provavelmente saltará dos 13 milhões de usuários em 2000, para 27 milhões pessoas nos Estados Unidos da América.

Do ponto de vista da legislação e dos direitos das pessoas idosas, o Estatuto do Idoso e a Política Nacional do Idoso (PNI), criada por meio da Lei n. 8.842/1994 e regulamentada pelo Decreto n. 1.948/1996, podem ser citados como aparatos que defendem os direitos aos idosos, o que inclui a convivência social, os cuidados e a manutenção da autonomia.

\section{SOBRE OS GRUPOS DE PARTILHA DE EXPERIÊNCIAS}

Quando há trocas entre iguais - portanto, entre pessoas que viveram experiências, perdas e papéis semelhantes -, o grupo pode tornar-se parte desta rede social significativa de seus participantes, o que resulta em ampliação da rede de suporte e na criação colaborativa de formas de enfrentamento de dificuldades (Abrahamsson et al., 2016; Grossi \& Santos, 2016; Rapizo \& Brito, 2014). Nos grupos de partilha entre pessoas com DP e entre familiares que cuidam, a expressão de sentimentos, os diálogos mediados sobre diagnósticos e prognósticos podem gerar formas diferentes de enfrentá-los e de viver o luto (Denborough, 2011). No caso de grupos com cuidadores, além de expressão de suas dificuldades e angústias e da construção de soluções para o seu dia a dia e para o enfrentamento dos impactos da DP para a pessoa de quem cuidam e seus familiares, os cuidadores se fortalecem para fazer frente a uma importante tarefa: ser a ou uma das mais importantes pessoas da rede de suporte de alguém que recebeu o diagnóstico da DP e cujos sintomas progressivamente reduziram ou reduzirão a qualidade de vida e autonomia. Por outro lado, quando a rede social significativa para a pessoa com DP fica muito restrita, o/a cuidador/a pode se tornar uma dentre as poucas pessoas com as quais a pessoa com DP convive e tem mais intimidade. Ambos, cuidador/a e pessoa com DP, devem evitar sobrecarregar tal relação para não aumentar os fatores de vulnerabilidade à saúde (Carmo \& Guizardi, 2018), o que foi observado nos relatos dos cuidadores e gerou a seguinte reflexão: os cuidadores de idosos, caso desenvolvam tal tarefa em tempo integral e descuidem dos próprios espaços de lazer, de aprendizado e de convivência podem ter também a sua rede de suporte 
e sua fonte de descanso, de restabelecimento e de trocas reduzida. O próprio grupo se mostrou um meio de ampliação de convivência e de amizades. Esgotamento do/da cuidador/a e violência contra idosos podem estar associados, daí a importância de que tenham, paralelamente, oportunidades de relação, reflexão, de pertencimento, de legitimação e de aprendizado conjunto, o que resulta em pausa na interação constante e exaustiva que podem viver e abertura para novas relações e trocas (Grossi \& Santos, 2016). Alinhado a tais reflexões, o grupo, além de partilha de informações e de experiências, conforme idealizado, pode ser um espaço de reflexão, o que ocorreu na medida em que temas foram escolhidos pelos participantes, que muito centraram suas falas em dificuldades no cuidar e nas relações.

\section{A DOENÇA DE PARKINSON}

Aproximadamente 1\% da população mundial com idade superior a 65 anos tem a doença, que atinge 200 mil pessoas no Brasil (Ministério da Saúde, 2017), com estimativa de que afete 8 milhões de indivíduos no mundo em 2030 e exija políticas de saúde, adaptação de vida e cuidados (Fernandes \& Andrade, 2018; WHO, 2006). A DP é uma doença neurodegenerativa progressiva e crônica, que não apresenta cura e cujos sintomas mais conhecidos são rigidez, acinesia (falta ou ausência de movimento), bradicinesia (lentificação dos movimentos), tremor de repouso e instabilidade postural (O'Sullivan \& Schimitz, 2004). Ainda, a literatura apresenta um elevado número de idosos com DP com sintomas de ansiedade e de depressão, em comparação à população em geral (Fernandes \& Andrade, 2018).

Devido aos sintomas apresentados acima, a maioria dos idosos com DP necessita de cuidadores. Os idosos com DP, maioria dos participantes do projeto, apresentam mais dificuldades para realizar atividades diárias simples, como trocar de roupa, segurar objetos etc. A dificuldade motora causada pela DP faz com que o paciente necessite de supervisão e auxílio durante certas tarefas. Ainda, idosos com DP apresentam um alto índice de quedas. Uma recente revisão mostrou que entre 35\% a $90 \%$ dos idosos com DP caem pelo menos uma vez (Allen, Schwarzel, \& Canning, 2013). As quedas reduzem significativamente a qualidade de vida de idosos com DP, a mobilidade e a expectativa de vida (Fausto, Canning, Hausdorff, Lord, \& Rochester, 2017) e são mais frequentes em idosas institucionalizadas (Soares \& Rech, 2015). Desta forma, a importância de um/a cuidador/a para idosos com DP aumenta significativamente.

\section{O PROJETO ATIVA PARKINSON}

O objetivo geral do projeto de extensão é oferecer um programa sistematizado de exercício físico, inter e transdisciplinar, para pessoas com DP. Especificamente, os objetivos são propiciar melhora na qualidade de vida de pessoas com DP (portanto, no quadro clínico), por meio da integração entre atividades físicas e sociais, e no tratamento farmacológico desenvolvido pelo neurologista responsável pelo paciente. Resumidamente, o projeto visa à colaboração com o desenvolvimento físico, psíquico e social, relacionado aos aspectos motores e não motores da doença, de pessoas com DP, estimulando a prática de atividade física grupal como parte integrante das atividades regulares dessa população. 
Profissionais e estudantes de educação física, fisioterapia, fonoaudiologia, psicologia, design, terapia ocupacional, enfermagem, dentre outros, propõem meios de tratamento e de cuidados com os pacientes e seus familiares (o que se identifica como ações multidisciplinares), mas conduzem o projeto conjuntamente, com foco na construção de um programa transdisciplinar voltado ao objetivo comum de propiciar qualidade de vida aos participantes - portanto, de manutenção de sua autonomia em níveis seguros. O trabalho dialogado, em que todos os campos do saber são privilegiados e seus conhecimentos utilizados em prol de objetivos comuns, aqui se identifica como transdisciplinaridade.

As atividades físicas em grupo são desenvolvidas duas vezes por semana, com duração de uma hora cada sessão. As sessões constam de três momentos: aquecimento/alongamento inicial (10 min.), parte principal (40 min.) e volta à calma/alongamento final (10 min.). Tanto as atividades físicas, lúdicas e de integração grupais, quanto as avaliações e pesquisas são discutidas no grupo de profissionais do projeto para que, além da integração e desenvolvimento social dos participantes, haja alinhamento e integração da equipe.

\section{O GRUPO DE PARTILHA COM CUIDADORES}

No Projeto Ativa, além das atividades realizadas com as pessoas com DP, são conduzidos, quinzenalmente, grupos de partilha com cuidadores, com o propósito de trocarem informações, dificuldades, sentimentos e necessidades relacionados ao cuidado e à convivência com a pessoa com DP. Têm participado dos grupos aproximadamente 10 cuidadores, sendo a maioria familiares: maridos, esposas, filhas e noras. Poderiam participar cuidadores profissionais que também necessitam ter claro o seu papel no cuidado da pessoa com DP, o progresso da doença, os sintomas motores e não motores e as diferenças individuais relacionadas aos sintomas e ao enfrentamento da doença, mas os cuidadores que se apresentaram são familiares.

Os cuidadores se reúnem em outra sala, mas no mesmo horário em que as pessoas com DP por eles cuidadas fazem atividades físicas grupais. Nos oito primeiros meses de 2019, o grupo de partilha foi mediado por duas extensionistas graduandas do Curso de Psicologia, da UNESP, supervisionadas por professora do mesmo curso.

Quando convidados para conversar em grupo sobre a experiência de cuidar, os cuidadores foram informados de que poderiam escolher sobre o que dialogar. Regularmente o grupo inicia com falas de acolhimento e que favoreçam a socialização e a partilha de fatos importantes do período. Caso em tais diálogos iniciais não surja um tema urgente, é proposta a discussão sobre um tema planejado de acordo com as indicações do grupo, frequentemente relacionado à importância de cuidar e aos impactos do cuidado na vida do/da cuidador/a, da pessoa com DP e da família.

Os mediadores do diálogo fazem perguntas e afirmações que geram reflexões, ampliação e busca por informações fidedignas, bem como oportunidades de autoconhecimento, de trocas afetivas, de expressão de sentimentos e de legitimação de pessoas e suas vivências. $O$ aprendizado conjunto e a criação de soluções para as dificuldades diárias pelo grupo são estimulados e enfatizados. Apesar de o grupo ter sido planejado pelos extensionistas como um espaço de conversas sobre experiências e informações, os participantes foram orientados desde o início que deveriam falar de si e não pelos outros, que conselhos deveriam ser desestimulados, 
nos moldes da Terapia Comunitária Sistêmica Integrativa (Grandesso \& Barreto, 2007), das Práticas Narrativas, Apreciativas e Colaborativas em que a valorização do outro e das diferenças são priorizadas (Cooperrider, Sorensen, Yaeger, \& Whitney, 2005; Denborough, 2011).

No grupo de partilha com cuidadores, reflexões têm sido expressas, são frequentemente incentivadas e há, portanto, diálogos acerca do diagnóstico, sintomas e tratamentos adequados à pessoa com DP, e também sobre o significado pessoal do cuidar; dificuldades e recursos do/da cuidador/a; qualidade de vida, saúde e lazer dos cuidadores e das pessoas com DP; relações familiares e relações cuidador/a-pessoa com DP; adaptações para segurança em casa; entre outros temas.

Os objetivos do grupo, de acordo com as bases epistemológicas construtivistas e teórica sistêmica integrativa, são fortalecer os cuidadores e seus vínculos, por meio de espaços de reflexão e de troca de informações que promovam e ampliem a sua autonomia, participação e qualidade de vida, o que tende a beneficiar o/a cuidador/a e também a pessoa de quem cuida (Amaral, Guazzelli, \& Feijó, 2017; Grandesso \& Barreto, 2007). O propósito de construção de conhecimento pelo grupo e de estímulo à troca e à busca fidedigna de informações pelos participantes é central, sem que os mediadores se omitam quando há dúvidas ou dados equivocados sobre a doença, seus sintomas, tratamentos e prognóstico. A segurança da pessoa com DP e a eficácia dos cuidados também são objetivos dos grupos de partilha. A Tabela 1 apresenta o resumo dos principais temas discutidos, conforme lista formulada pelos participantes nos primeiros encontros e ampliada no decorrer do trabalho. 
Tabela 1: Temas abordados no Grupo de Partilha de Cuidadores de Pessoas com Doença de Parkinson.

\begin{tabular}{|c|c|}
\hline TEMAS & RESUMO \\
\hline $\begin{array}{l}\text { Cuidando do/ } \\
\text { da cuidador/a }\end{array}$ & $\begin{array}{c}\text { Buscou-se compreender qual a importância do "cuidar" para os participantes } \\
\text { do grupo, para então introduzir a discussão sobre os limites que cuidar } \\
\text { do outro possui, as implicações na saúde do/da cuidador/a e como isso } \\
\text { pode ser gerido de forma prática no dia a dia. }\end{array}$ \\
\hline $\begin{array}{l}\text { Redes de } \\
\text { apoio }\end{array}$ & $\begin{array}{l}\text { Nesta atividade, as mediadoras procuraram evidenciar a importância da rede de } \\
\text { apoio dos cuidadores. Em partilha, nasceu uma metáfora sobre a sustentação de uma } \\
\text { casa. Ela (a casa), apesar de ter uma coluna principal, necessita que outras suportem } \\
\text { o peso da construção. Da mesma forma, o/a cuidador/a necessita que outros } \\
\text { cuidadores (secundários) auxiliem na tarefa de cuidar, para que a sobrecarga não } \\
\text { prejudique a qualidade de vida do/da cuidador/a e do próprio cuidado oferecido. } \\
\text { Foi abordada também a necessidade de apoio tanto da família, quanto amigos } \\
\text { e pessoas que partilham de contextos semelhantes. }\end{array}$ \\
\hline Medicação & $\begin{array}{l}\text { Com o auxílio das mediadoras da área de psicologia e enfermagem, conversou-se } \\
\text { a respeito de possíveis efeitos colaterais da medicação de Doença de Parkinson } \\
\text { e houve o esclarecimento de tratamentos alternativos que vêm surgindo à DP. } \\
\text { Além disso, discutiu-se formas de administrar tais medicamentos. }\end{array}$ \\
\hline $\begin{array}{l}\text { Autonomia/ } \\
\text { liberdade }\end{array}$ & $\begin{array}{l}\text { Compartilhar as limitações que envolvem ser o/a cuidador/a de alguém com DP foi } \\
\text { o gatilho usado para discutir-se a relevância de promover comportamentos } \\
\text { autônomos dos indivíduos com DP, como forma de reduzir a sobrecarga do/da } \\
\text { cuidador/a, bem como promover independência e melhor qualidade de vida do } \\
\text { paciente, ao se sentir capaz de realizar tarefas, mesmo que de modo adaptado. } \\
\text { Em suma, tal atividade visou ao incentivo dos/as cuidadores a promoverem } \\
\text { comportamentos independentes dos pacientes que não os coloquem em risco. }\end{array}$ \\
\hline Alimentação & $\begin{array}{l}\text { Houve o esclarecimento de dúvidas sobre a dieta recomendada aos pacientes com DP, } \\
\text { por meio de explicações sobre o funcionamento/reação do organismo com alguns } \\
\text { alimentos e o medicamento necessário ao tratamento da DP. A discussão também } \\
\text { envolveu aspectos relacionados a como o/a cuidador/a se encontra nessa situação: } \\
\text { se também se adaptou à dieta e se há a privação para colaborar com o outro. }\end{array}$ \\
\hline Casa segura & $\begin{array}{l}\text { Mediado pelas voluntárias de enfermagem, o tema Casa Segura foi sugerido } \\
\text { pelos participantes para que se partilhassem dicas/recomendações práticas para } \\
\text { evitar quedas, como, por exemplo, colocar fita nos tapetes para que esses não } \\
\text { deslizem e provoquem quedas. }\end{array}$ \\
\hline Tempo & $\begin{array}{l}\text { Foi enfatizada a relação entre tempo, desempenhado e a tensão relacionada } \\
\text { a ser cuidador/a, por meio da valorização de atividades que promovam } \\
\text { o bem-estar dos/das cuidadores/as. Buscou-se, então, fazer um levantamento } \\
\text { de atividades prazerosas para os/as cuidadores/as e estratégias de gestão de tempo, } \\
\text { mediante a disponibilização de uma ferramenta de cronograma semanal, } \\
\text { como auxílio possível para administração de tarefas. }\end{array}$ \\
\hline Luto & $\begin{array}{l}\text { O tema foi abordado a fim de desmistificar o sentido comum de luto, para que } \\
\text { os participantes compreendessem o luto como "perdas simbólicas" decorrentes } \\
\text { de várias situações (perda de funções que desempenhavam, sonhos, papéis etc.). } \\
\text { Além disso, houve diálogo sobre sentimentos e adaptações exigidas após as } \\
\text { perdas; como agem e o que poderia ser feito. }\end{array}$ \\
\hline Compartilhar & $\begin{array}{l}\text { Foi desenvolvida discussão sobre a importância de compartilhar tanto experiências } \\
\text { boas quanto ruins, enfatizando o papel da fala no processo de melhora na tensão } \\
\text { ocasionada em determinadas situações. O tema se expandiu para além de assuntos } \\
\text { relacionados à DP, compreendendo as diversas posições em que os participantes se } \\
\text { encontram como cuidadores/as e ponto de referência para família e amigos. }\end{array}$ \\
\hline
\end{tabular}




\section{PRINCIPAIS RESULTADOS}

A participação assídua e engajada dos/as cuidadores/as de pessoas com DP ao longo do ano foi um importante resultado das atividades reflexivas em grupo. Soluções construídas pelos participantes para minimizar a sobrecarga relacionada aos cuidados e intensa convivência com a pessoa com DP e suas perdas consecutivas também apontaram para a relevância do trabalho. O grupo de cuidadores/as decidiu caminhar em um período no qual as pessoas com DP estavam em atividades com a equipe do projeto. Após tal iniciativa, foi-lhe renovado o convite para participar de atividades lúdicas grupais com as pessoas com DP, sem que ficassem ao lado delas para protegê-las, já que aquele era um momento em que a equipe do projeto se responsabilizava pelo cuidado das pessoas com DP. Neste sentido, além de reflexões, o grupo de partilha gerou ações de integração entre cuidadores/as e entre os dois grupos: cuidadores/as e pessoas com DP.

Os/as cuidadores/as se conectaram por aplicativo de mensagens, comemoraram resultados de tratamentos e dialogaram sobre a participação no projeto por meio de mensagens. Além disso, utilizaram os celulares para emitir informações relevantes ao cuidado com as pessoas com DP para as responsáveis pela mediação do grupo. Internações, quedas, sintomas e outras questões foram apontadas, o que ampliou a possibilidade de suporte social.

\section{AS PESQUISAS}

As ações de extensão, resumidamente relatadas no artigo, têm alimentado, além de novos projetos, novas pesquisas dos autores e de parceiros, o que compreendemos ser ao mesmo tempo um resultado e um cuidado com o embasamento das práticas. Pesquisas sobre inclusão e suporte social vêm sendo realizadas por pesquisadores da Universidade $\mathrm{x}$ em parceria com outras universidades. Pessoas com deficiências congênitas ou adquiridas e seus familiares foram entrevistados por Silva (2020), que destacou a importância do apoio familiar à inclusão de Pessoas com Deficiência (PcDs). Tal resultado de pesquisa se alinha à proposta de grupos de partilha com cuidadores/as, especialmente por enfatizar que a visão positiva sobre a inclusão é importante, mas não basta. Cuidadores precisam ser orientados sobre como estimular a autonomia, sem supervalorizar as possibilidades de quem cuida e sem superprotegê-las. A reflexão gerada pelos grupos de partilha, sustentada em pesquisa e enfocada no presente artigo é de que, além de trocas e de orientação, o grupo deve ser estimulado a criar a própria pauta e a refletir sobre suas demandas, necessidades, forças e conquistas.

Bianca Augusto (2019), que atua como mediadora do Grupo de Partilha com cuidadores, realizou paralelamente, à prática de extensão, pesquisa em que entrevistou profissionais das áreas de educação física, fisioterapia, terapia ocupacional, psicologia e neurologia, para compreender que informações devem ser repassadas aos cuidadores/as de pessoas com DP (Augusto \& Feijó, 2019). Os entrevistados ressaltaram, entre outros aspectos, as possíveis perdas cognitivas e de memória decorrentes da DP, que não são amplamente conhecidas. Enfatizaram a importância de cuidadores/as promoverem a autonomia, sem descuidar das situações que podem trazer vulnerabilidade à pessoa com $\mathrm{DP}$, que em estágio avançado pode esquecer o caminho de casa, por exemplo. Os entrevistados mencionaram a relevância do suporte familiar e trataram de efeitos da medicação e da importância de familiares de pessoas com DP falarem abertamente, aos profissionais, 
sobre eventuais sonhos, manias, desejos exacerbados que podem aparecer em decorrência de uso de certos medicamentos por pessoas com DP. Os encontros de grupos de cuidadores e de pessoas com DP podem ser momentos especiais para reflexão sobre comunalidades e especificidades, com potencial de maior compreensão de certas vivências e comportamentos.

A atuação multidisciplinar e em equipe que compartilha objetivos comuns (transdisciplinaridade) também foi apontada como benéfica por muitos participantes da pesquisa. Além disso, a relação cuidador/a-familiares foi mencionada como relevante. Os resultados das referidas pesquisas reforçam a importância dos grupos de partilha, na medida em que nesses é possível trocar informações sobre a DP, tratamentos, sintomas e recursos de cuidado, além de acolher cuidadores/as que precisam de suporte para a manutenção do apoio à pessoa com DP.

Gaspar e Hernandes (2016) realizaram estudo qualitativo descritivo sobre mudanças ocorridas na rede social de idosos, após sua entrada em uma Instituição de Longa Permanência (ILPI). Foram entrevistadas 12 pessoas acima de 60 anos de idade, de ambos os sexos, que apresentaram funções mentais preservadas. Os dados sobre aspectos estruturais e funcionais da rede social pessoal, antes e após a institucionalização do idoso, mostraram que muitos idosos viviam sozinhos ou com poucos contatos com amigos e familiares antes da internação e, portanto, apresentaram aumento da rede social com o estabelecimento de novas amizades na ILPIs e com ampliação das relações comunitárias. Cabe ressaltar que a maioria dos participantes tinha dificuldade de acesso à renda. Apesar de tais resultados positivos do ponto de vista do tamanho da rede após institucionalização, as relações íntimas, tão importantes para a legitimação, autoestima e pertencimento, afetam o bem-estar e a saúde quando escassas. Sendo assim, a institucionalização deve ser evitada, a menos que a pessoa com DP não tenha quem dela cuide e viva isolada. No projeto do qual faz parte o grupo de partilha com cuidadores, com previsão de início do grupo de partilha com pessoas com DP, os participantes vivem com suas famílias e frequentam o projeto na universidade, onde encontram grupos de iguais, além de diversos alunos e profissionais. Os grupos de partilha são uma parte de uma ação maior de cuidado e de manutenção de rede social de pessoas com diferentes condições socioeconômicas.

A proposta de reflexão nos grupos, do cuidado com o/a cuidador/a e com a relação entre este e a pessoa com DP, tal como apresentada no artigo, também vem ao encontro das observações da pesquisa, na medida em que principalmente os/as cuidadores/as familiares devem receber atenção, o que contribuirá para a manutenção de relações familiares e íntimas, que são insubstituíveis em certos aspectos. Pessoas que viveram histórias juntas e que construíram cumplicidade são importantes fontes de suporte social (Sluzki, 1997).

Além das pesquisas que indicam a relevância do suporte social aos idosos e aos cuidadores/as de idosos e de pessoas com doenças, dados epidemiológicos e projeções demográficas evidenciam a necessidade de políticas e de práticas adequadas à manutenção da qualidade de vida de pessoas com mais de 60 anos e de pessoas com deficiências. A população idosa cresce vertiginosamente no país e no mundo, conforme aponta o IBGE (2010) e foi apontado no início deste artigo. 


\section{CONSIDERAÇÕES FINAIS}

De acordo com autores sistêmicos, a rede social é importante fonte de suporte, de socialização e de legitimação, e a boa qualidade de relação com pessoas e instituições que formam a rede social de suporte protege a saúde.

As pessoas que, por idade avançada, adoecimento, dificuldades de locomoção, condição socioeconômica ou necessidade de cuidar de outrem em tempo integral têm parte da sua rede de suporte reduzida, se encontram vulneráveis por apresentarem redução das relações sociais, o que pode resultar em desgaste emocional e em adoecimento, com manifestações físicas, psíquicas e sociais. As ações do projeto de extensão mostraram que tanto as pessoas com DP, que neste caso são idosas, quanto seus cuidadores/as, também idosos em sua maioria, reduziram a convivência com outras pessoas - fora do projeto em questão. Sendo assim, é importante que pessoas idosas ou jovens com DP e seus/suas cuidadores/as mantenham o convívio com pessoas, principalmente aquelas que com eles compartilham histórias e dificuldades semelhantes: familiares, amigos, outras pessoas com DP e outros/as cuidadores/as. Além do convívio e da oportunidade de expressão de angústias, forças e conhecimentos, podem falar sobre suas vidas, perspectivas, habilidades, sonhos e planos.

A experiência com os grupos mostrou que, para os/as cuidadores/as e para as pessoas com DP, é importante ter planos realistas, mas, para tal, precisam ter conversado, com suporte e informações claras, sobre as possibilidades de avanço da DP. A morte não precisa se manter em foco, mas o/a cuidador/a que abandona seu desenvolvimento e projetos, para se dedicar por longo período aos cuidados da pessoa com DP, pode ter sua vida esvaziada de propósitos quando encerra a sua atividade de cuidador/a. Se for um cuidador/a profissional, deve ter a rede de encaminhamento de outros clientes mantida e atualizados seus conhecimentos como cuidador/a, além de cuidado com sua própria saúde (alimentação, sono, exercícios físicos, lazer, convivência familiar). Já o/a cuidador/a que também é da família, além dos cuidados com a própria saúde e desenvolvimento, precisa manter ativa a rede de amizades, o contato e o bom relacionamento com outros familiares, resolvendo conflitos interpessoais de forma pacífica e dialogada. Tal observação é fruto de diálogos recorrentes no grupo, sobre o relacionamento familiar, especialmente de quem cuida com quem é cuidado. O enrijecimento de papel, em que só uma pessoa da família se encarrega dos cuidados, sem que outras colaborem e dividam responsabilidades, adoece o/a cuidador/a principal, parte da família, e piora a qualidade de vida da pessoa com DP. Dessa forma, além das atividades externas para a pessoa com DP e para o/a cuidador/a, em alguns casos, relações familiares precisam ser cuidadas.

$\mathrm{Na}$ sequência da conexão entre as atividades de extensão e de pesquisa sintetizadas, cabe ressaltar que, como toda a deficiência adquirida, o diagnóstico da DP e os sintomas e disfuncionalidades que são desenvolvidos geram um forte impacto na pessoa com DP, no/na cuidador/a e na família. Os cuidados psicológicos são, portanto, frequentemente necessários para que as pessoas lidem com as perdas e com o luto da perda de parte da autonomia, sem abandonar a qualidade de vida que ainda lhes resta ${ }^{1}$.

As demandas por realização de um grupo com as pessoas com DP e do incremento de estímulo à reflexão foram observadas por ocasião da escrita do presente artigo, que também possibilitou que fosse planejada a futura ampliação do estudo, com entrevistas com os/as cuidadores/as e com as pessoas com DP sobre os benefícios da participação no projeto e nos encontros, que atualmente são relatadas sem registro em áudio.
10 processo de publicação do artigo ocorreu em parte nos meses de abril e maio de 2020, em que a pandemia da COVID-19 afetou sobremaneira o Brasil. A equipe do projeto de extensão, cuja ação é alvo de reflexão, preocupada com mais uma redução de meios de acesso à rede social dos participantes, tem mantido contato com pessoas com DP e cuidadores por telefone e aplicativo de mensagens. 


\section{REFERÊNCIAS}

Abrahamsson, C., Rangel, C. L., Ribeiro, C. F., Costa, D. M., Beuque, F. M. V. D., Costa, H. R. et al. (2016). Ateliês em curso: Tecendo metodologias de facilitação. Nova Perspectiva Sistêmica, 25(56), 22-33. Recuperado de https://www.revistanps. com.br/nps/article/view/238.

Allen, N., Schwarzel, A., \& Canning, C.G. (2013). Recurrent Falls in Parkinson's Disease: A Systematic Review. Parkinson's Disease, 2013, 1-16. https://doi. org/10.1155/2013/906274 . Recuperado em https://www.hindawi.com/journals/ $\mathrm{pd} / 2013 / 906274 /$

Amaral, C. B., Guazzelli, J. K., \& Feijó, M. R. (2017). Grupo Reflexivo sobre qualidade de vida em uma instituição de longa permanência para idosos. In H. F. Cardoso, M. R. Feijó, M. L. Camargo, D. C. Campos, \& E. Goulart (Orgs.), Experiências de Formação em Psicologia Organizacional e do Trabalho e Orientação Profissional (pp. 99-110). Araraquara, SP: Letraria.

Augusto, B. S. \& Feijó, M. R. (2019). Contribuições para uma Capacitação Multidisciplinar de Cuidadores de Pessoas com Doença de Parkinson. Trabalho apresentado no XXXI Congresso de Iniciação Científica da Unesp. Recuperado de file://C:/Users/usuario/Downloads/RESUMO_44750810843_ptg\%20(1).pdf

Brito, T. R. P \& Pavarini, S. C. I. (2012). Relação entre apoio social e capacidade funcional de idosos com alterações cognitivas. Rev. Latino-Am. Enfermagem, 20(4), 677-684. doi: 10.1590/S0104-11692012000400007.

Camargo, M. L. \& Feijó, M. R. (Orgs.). (2017). Inclusão de pessoas com deficiência no trabalho: orientações para organizações contratantes. Araraquara, SP: Letraria.

Carmo, M. \& Guizardi, F. (2018). O Conceito de vulnerabilidade e seus sentidos para as políticas públicas de saúde e assistência social. Cadernos de Saúde Pública, 34(3), e00101417. Epub March 26, 2018. doi:10.1590/0102-311x00101417.

Cooperrider, D. L., Sorensen, P. F. Jr., Yaeger, T. F., \& Whitney, D. (2005). Appreciative Inquiry: Foundations in Positive Organization Development. Champaign, IL: Stipes Publishing.

Couto, M. C. P. P., Koller, S. H., \& Novo, R. F. (2006). Resiliência no Envelhecimento: Risco e Proteção. In D. V. S. Falcão \& C. M. S. B. Dias (Orgs.), Maturidade e Velhice: pesquisas e intervenções psicológicas (pp. 315-337). São Paulo: Casa do Psicólogo.

Denborough, D. (2011). Atendendo crianças que vivenciaram traumas: a árvore da vida. Nova Perspectiva Sistêmica, 20(39), 92-101. Recuperado de http://www. revistanps.com.br/nps/article/view/192/174.

Dias, D. S. G., Carvalho, C. S., \& Araújo, C. V. (2013). Comparação da percepção subjetiva de qualidade de vida e bem-estar de idosos que vivem sozinhos, com a família e institucionalizados. Revista Brasileira de Geriatria e Gerontologia, 16(1), 127-138. doi: 10.1590/S1809-98232013000100013.

Fausto, A., Canning, C. G., Hausdorff, J. M., Lord, S., \& Rochester, L. (2017). Falls in Parkinson's disease: A complex and evolving picture. Recuperado de https://onlinelibrary.wiley.com/doi/full/10.1002/mds.27195.

Feijó, M. R. (2006). Família e Rede Social. In C. M. O. Cerveny (Org.), Família e narrativas, gênero, parentalidade, irmãos, filhos nos divórcios, genealogia, história, estrutura, violência, intervenção sistêmica, rede social (pp. 236-253). São Paulo: Casa do Psicólogo.

Fernandes, I. \& Andrade, A. (2018). Estudo clínico-epidemiológico de pacientes com Doença de Parkinson em Salvador-Bahia. Revista Brasileira de Neurologia e Psiquiatria, 22(1), 45-59. Recuperado de https://www.revneuropsiq.com.br/ rbnp/article/view/244. 
Friedland, R. (2019). What is Long-Term Care? In Family Caregiver Alliance. Selected Long-Term Care Statistics. Recuperado de https://www.caregiver.org/ selected-long-term-care-statistics.

Gaspar, C. \& Hernandes, E. (2016). Rede Social de Idosos Institucionalizados. Curso de Especialização em Intervenção Familiar: Psicoterapia e Orientação Sistêmica. São José do Rio Preto: Faculdade de Medicina de São José do Rio Preto - FAMERP.

Giacomin, K. C. (2016). Considerações Finais. In A. O. Alcântara, A. A. Camarano \& K. C. Giacomin (Orgs.), Política nacional do idoso: velhas e novas questões (pp.593-615). Rio de Janeiro: Ipea. Recuperado de http://www.ipea.gov.br/portal/ images/stories/PDFs/livros/livros/161006_livro_politica_nacional_idosos.PDF.

Grandesso, M. \& Barreto, M. (2007). Terapia Comunitária. Tecendo redes para a transformação social, saúde, educação e políticas públicas. São Paulo: Casa do Psicólogo.

Grossi, P. K. \& Santos, A. M. (2016). Envelhecimento e Cuidados. Relatos de experiências com cuidadores de pessoas idosas. Porto Alegre: EDIPUCRS.

Horta, A. L. \& Daspett, C. (2007) Terapia Comunitária como instrumento de diagnóstico de saúde e de funcionários do PSF da Zona Norte de São Paulo. In M. Grandesso \& M. Barreto (Orgs.), Terapia Comunitária. Tecendo redes para a transformação social, saúde, educação e políticas públicas (pp. 387-390). São Paulo: Casa do Psicólogo.

Instituto Brasileiro de Geografia e Estatística - IBGE. (2019). Síntese de indicadores sociais: uma análise das condições de vida da população brasileira. Recuperado de https://biblioteca.ibge.gov.br/visualizacao/livros/liv98965.pdf.

Lei n. 8.842, de 04 de janeilro de 1994. (1994). Dispõe sobre a Política Nacional do Idoso, cria o Conselho Nacional do Idoso e dá outras providências. Recuperado de http://www.planalto.gov.br/ccivil_03/leis/18842.htm

Marinho, S. M. B. A. (2010). O suporte social e a depressão no idoso. Dissertação de Mestrado, Programa de Pós-graduação em Promoção da Saúde, Faculdade de Ciências Humanas e Sociais, Universidade Fernando Pessoa, Porto, Portugal.

Ministério da Saúde. (2017). Pacientes com Parkinson contarão com novos medicamentos no SUS. Brasília, 2017. Recuperado de http://portalms.saude. gov.br/noticias/agencia-saude/41873-pacientes-com-parkinsoncontarao-comnovos-medicamentos-no-sus.

Moretto, G. F., Rodrigues, S., Polastri, Pn, Fiorelli, C.M., Costa, E. D., Gottardi, G. C., Vieira, L. B. et al. (2015). Ativa Parkinson: benefícios da atividade física na qualidade de vida de pessoas com doença de Parkinson. Recuperado de https://repositorio. unesp.br/handle/11449/142101

0'Sullivan, S. B. \& Schimitz, T. J. (2004). Doença de Parkinson. In Fisioterapia: avaliação e tratamento (pp. 747-73). São Paulo: Manole.

Rapizo, R. \& Brito, L.M.T. (2014). Espaço de conversas sobre o divórcio: a diferença como recurso para transformação. Nova Perspectiva Sistêmica, 23(50), 32-50. Recuperado de https://www.revistanps.com.br/nps/article/view/90.

Rizzolli, D. \& Surdi, A. C. (2010). Percepção dos idosos sobre grupos de terceira idade. Rev. Bras. Geriatr. Gerontol., 13(2), 225-233. ISSN 1809-9823. https://doi. org/10.1590/S1809-98232010000200007 . Recuperado de https://www.scielo.br/ scielo.php?pid=S1809-98232010000200007\&script=sci_abstract\&tlng=pt

Saintrain, M. V. L, Bandeira, C. B., Nobre, M. A., \& Sandrin, R. L. P. (2018). Idosos com Depressão: uma análise dos fatores de institucionalização e apoio familiar. Revista Brasileira em Promoção da Saúde, 31(4), 1-7. DOI: http://dx.doi. org/10.5020/18061230.2018.8763. Recuperado de https://www.scielo.br/scielo. php?pid=S1809-98232010000200007\&script $=$ sci_abstract\&tlng $=$ pt

Nova Perspectiva Sistêmica, v. 29, n. 68, p. 31-45, dezembro 2020. 
Silva, A. (2020). Orientação profissional: percepção e participação familiar no processo de escolha profissional de pessoas com deficiência. Dissertação de Mestrado, Programa de Pós-graduação em Psicologia do Desenvolvimento e da Aprendizagem. Faculdade de Ciências, Universidade Estadual Paulista, Bauru, SP.

Sluzki, C. E. (1997). A rede social na prática sistêmica: alternativas terapêuticas. São Paulo: Casa do Psicólogo.

Soares, I. G. E. \& Rech, V. (2015) Prevalência de quedas em idosos institucionalizados no Brasil: uma revisão integrativa. Revista Kairós Gerontologia, 18(4), 47-61. ISS Ne 2176-901X. Recuperado de https://revistas.pucsp.br/index.php/kairos/article/ viewFile/26892/19101

Vitorino, L. M., Paskulin, L. M. G., \& Vianna, L. A. C. (2013). Qualidade de vida de idosos da comunidade e de instituições de longa permanência: estudo comparativo. Revista Latino-Americana de Enfermagem, 21(nspe.), 3-11. https:// doi.org/10.1590/S0104-11692013000700002. Recuperado de www.revistas.usp. $\mathrm{br} / \mathrm{rlae} / \mathrm{article} / \mathrm{view} / 52920$

World Health Organization - WHO. (2006). Global Burden of Neurological Disorders: estimates and projections In Neurological disorders: public health challenges, chapter_2, 27-39. ISBN 924156336 2. Recuperado de https://www.who.int/ mental_health/neurology/chapter_2_neuro_disorders_public_h_challenges. pdf?ua $=1$. 


\section{MARIANNE RAMOS FEIJÓ}

Professora assistente, doutora da Universidade Estadual Paulista (UNESP) Faculdade de Ciências - Departamento de Psicologia (Bauru, SP) e professora na pós-graduação em Psicologia do Desenvolvimento e da Aprendizagem na Unesp. Psicóloga, especialista em Terapia Familiar e de Casal, mestre e doutora em Psicologia Clínica (PUC-SP), pós-doutora em Psicobiologia (UNIFESP). https://orcid.org/0000-0002-6036-9884

E-mail: marianne.r.feijo@unesp.br

\section{FABIO AUGUSTO BARBIERI}

Professor assistente, doutor da Universidade Estadual Paulista (UNESP) - Faculdade de Ciências - Departamento de Educação Física (Bauru, SP) e professor do Programa de Pós-Graduação em Ciências da Motricidade-Interunidades na UNESP (Capes 5). Bacharel em Educação Física (2004) e mestre em Ciências da Motricidade (2007) pela UNESP. Doutor em Ciências da Motricidade pela UNESP (2012) e Ph.D. em Human Movement Science pela VU Amsterdam University (Holanda - 2013). Pós-doutor em Educação Física pela UNESP.

https://orcid.org/0000-0002-3678-8456

E-mail: fabio.barbieri@unesp.br

\section{TAFNES IKEGAMI PEREIRA}

Graduanda em Psicologia na Universidade Estadual Paulista - UNESP Bauru, SP; organizadora do Grupo de Partilha de Cuidadores de Pessoas com Doença de Parkinson no projeto Ativa Parkinson do Laboratório de Pesquisa em Movimento Humano (MOVI-LAB) e bolsista no Programa PET-Saúde/Interprofissionalidade. https://orcid.org/0000-0002-6245-1208

E-mail: tafnes.ikegami@unesp.com

\section{BIANCA SILVA AUGUSTO}

Graduanda em Psicologia na Universidade Estadual Paulista - UNESP Bauru, SP com experiência em grupos reflexivos com cuidadores de pessoas com Doença de Parkinson no projeto Ativa Parkinson do Laboratório de Pesquisa em Movimento Humano (MOVI-LAB).

https://orcid.org/0000-0003-3488-9189

E-mail: bianca.augusto@unesp.br

\section{CAROLINA SILVA GASPAR}

Psicóloga do TJSP - Tribunal de Justiça do Estado de São Paulo. Especialista em Intervenções Sistêmicas e Terapia Familiar pela FAMERP, São José do Rio Preto, SP. https://orcid.org/0000-0003-1842-8251

E-mail: carolina gaspar@hotmail.com

Nova Perspectiva Sistêmica, v. 29, n. 68, p. 31-45, dezembro 2020. 